\title{
Информационная оценка метода химического анализа
}

\author{
Шапошник B.A. \\ ФБГОУ ВО Воронежский государственный университет», Воронеж
}

Поступила в редакцию 6.03.2018 г.

DOI: https://doi.org/10.17308/sorpchrom.2018.18/491

Аналитическую химию отличает множественность вариантов анализа элементов и веществ. Теория информации даёт возможность выбрать метод анализа на основе сравнения скоростей, разрешающейся способности и воспроизводимости. В статье рассмотрено применение теории информации для сравнения ионной и классической ионообменной хроматографии. Важной характеристикой метода анализа является его рентабельность, определяемая как отношение количества информации к стоимости прибора и его обслуживания.

Ключевые слова: информация, скорость анализа, разрешение, воспроизводимость, рентабельность, хроматография.

\section{Information evaluation of the chemical analysis method}

\author{
Shaposhnik V.A. \\ Voronezh State University, Voronezh
}

Analytical chemistry is distinguished by a plurality of variants of the analysis of elements and substances. The article deals with the principle of choosing the most effective and cost-effective method of analysis. The objective of this publication is to present the stages of the analytical process from the point of information theory, discuss the possibility of quantitative evaluation of a particular method of analysis and comparative analysis of the analysis methods.

From the point of view of the information theory, the stages of the analytical process include the analysis of samples as a special case of the information source, the selection operations as a special case of the preliminary transformation, the measurement as a special case of coding, comparison with the standard as a special case of decoding. It is important to pay attention to the fact that at all stages of the transmission of information possible random errors, in the noise, and systematic errors. For the relative evaluation of the information of the two methods, we propose an equation in which the relative amount of information is a product of factors characterizing the comparison of the times of the two methods, their resolution and precision. As an example, examined the comparative information content of methods for the determination of cations of alkali metals in solution using ionic chromatography and classical ion exchange chromatography. The calculation of the ratio of the values of the information obtained by these methods gave a value of 4000 .

For a real assessment of the choice of an effective method of analysis, profitability should be calculated as the ratio of the amount of information to the cost of the device, reagents and others.

Keywords: information, speed of analysis, resolution, precision, profitability, chromatography.

\section{Введение}

Трудно назвать какое либо достижение в физике, химии и даже биологии, которое нельзя было бы использовать для анализа химических веществ. Дивергенция 
методов аналитической химии столь значительна, что создаёт проблему выбора оптимального метода анализа определенного вещества в определенных условиях. Предположим, что студенту, прошедшему университетский курс аналитической химии будет необходимо определить содержание ионов меди в растворе. Он вспомнит реакции иодометрического и комплексонометрического титрования, электроосаждение, вольтамперометрический метод, фотометрической определение, метод атомноабсорбционного анализа, ионной хроматографии. Однако такой перечень методов ничего не говорит о возможности выбора наиболее рационального метода определения меди, тем более, если лаборатория располагает возможностью использования не только перечисленные методы, но и множество других. Наиболее общим подходом к решению предлагаемой задачи будет поиск наиболее информативного метода. В настоящее время информационная оценка метода возможна только на основе общей теории информации.

Норберт Винер назвал информацию цементом, скрепляющим общество. Прошлый век имел много прилагательных, нынешний век обычно характеризуют преимущественно как информационный. Обмен информацией является задачей научных конференций и публикаций, неформальных дискуссий, которые создают научные сообщества. Даже биологическая эволюция может быть рассмотрена как увеличение информации [1]. Задачей настоящей публикации является рассмотрение стадий аналитического процесса с точки теории информации, обсуждение возможности количественной оценки информации конкретного метода анализа и сравнительный анализ информативности методов анализа на примере классической ионообменной и ионной хроматографии. Кроме того обсуждается возможность оценки рентабельности метода как отношения, содержащегося в нем количества информации к стоимости прибора, реагентов и его обслуживания.

\section{Стадии аналитического процесса}

С точки зрения теории информации стадии аналитического процесса включают отбор пробы как частный случай источника информации, операции выделения как частный случай предварительного преобразования, измерение как частный случай кодирования, сравнение с эталоном как частный случай декодирования. Важно обратить внимание на то, что на всех стадиях передачи информации возможны случайные ошибки, в общем случае шумы, и систематические ошибки, в общем случае помехи. Недостатком этих схем является отсутствие обратной связи полученной информации с начальными стадиями процесса, которое позволило бы применить принципы кибернетики к химическому анализу. В частности, это было бы полезно для высокоэффективной жидкостной хроматографии при использовании обратной связи для смешивания растворителей. Впервые на аналогию процесса передачи информации от источника до получателя со стадиями аналитического процесса обратил внимание X. Кайзер [2]. Рис. 1 демонстрирует аналогию информационного канала и аналитического процесса.

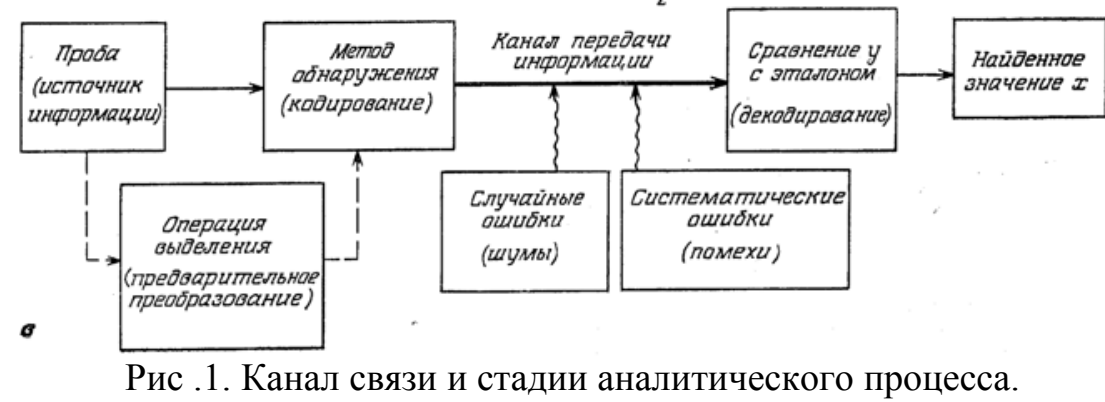

Шапошник B.A. / Сорбционные и хроматографические процессы. 2018. Т. 18. № 2 
Рассмотрим в качестве примера фотометрический метод анализа меди. Процесс начинается с отбора пробы, которая является источником информации. Если выбрать фотометрический метод, то для усиления абсорбции света в пробу вводят аммиак (предварительное преобразование) для увеличения интенсивности окраски полученного аммиаката меди. При фотометрическом измерении результат кодируется в виде величины оптической плотности. Для декодирования результата используется градуировка по стандартным растворам, которая даёт возможность определить концентрацию меди.

\section{Количественная оценка информации аналитического метода}

Количественный подход к оценке информации стал возможен на основе уравнения, предложенного в 1948 г. Клодом Шенноном [3]

$$
I=\sum_{i} p_{i} l d p_{i}
$$

где $I$ - количество информации, $p_{i}$ - вероятность того, что именно событие $i$ верно.

Теория Шеннона была применена к аналитической химии К. Дёрфелем, В. Хильдебрандтом, К. Экшлагером и К. Данцером [4-7]. Полученное уравнение имеет вид

$$
I_{i}=\frac{\tau_{o}}{\tau} \cdot \frac{\Delta x}{\Delta x_{1 / 2}} \cdot l d \frac{\Delta C \sqrt{n}}{2 s \cdot t},
$$

где $I_{i}$ - количество информации содержащейся в аналитическом сигнале, $\tau_{o}$ - время, необходимое для анализа, $\tau$ - фактическое время анализа, $\Delta x$ - интервал параметра развертки, $\Delta x_{1 / 2}$ - полуширина аналитического сигнала, $\Delta C$ - интервал измеряемой концентрации, $n$ - количество измерений, $s$ - стандартное отклонение, $t$ - квантиль распределения Стьюдента. Первый множитель выражает экспрессность анализа. Прорывы в аналитической химии характеризуются в первую очередь значительным увеличением экспрессности. Второй множитель характеризует разрешающую способность метода, третий - воспроизводимость анализа.

В связи с неопределенностью задания времени, необходимого для анализа, интервалов параметра развертки и интервала концентраций будем использовать отношение количества информации, заменив полуширину сигнала шириной его у основания, как это принято в хроматографии

$$
\frac{I_{1}}{I_{2}}=\frac{\tau_{2}}{\tau_{1}} \cdot \frac{W_{2}}{W_{1}} \cdot l d \frac{s_{2} t_{2}}{s_{1} t_{1}} \sqrt{\frac{n_{1}}{n_{2}}}
$$

В качестве примера сравним количества информации в классической ионообменной и ионной хроматографии. Рис. 2 показывает хроматографическое разделение смеси ионов щелочных металлов методом классической ионообменной хроматографии [8]. Для разделения смеси катионов требуется от 2 до 4 суток. Причиной малой скорости анализа является медленная сорбция и десорбция ионов при молекулярной диффузии в ионообменнике.

Ионная хроматография, предложенная Х. Смоллом, Т. Стивенсом и В. Бауманом в 1975 г. [9], представляет вариант высокоэффективной ионообменной хроматографией. Её отличием от классического варианта является использование модификации поверхности сорбента и высокоскоростное кондуктометрическое детектирование. Кроме того, применяется двухколоночный вариант, при котором увеличивается величина сигнала переводом солей щелочных металлов в их гидроксиды. Рис. 3 по- 
казывает разделение катионов щелочных металлов методом ионной хроматографии $[8]$.

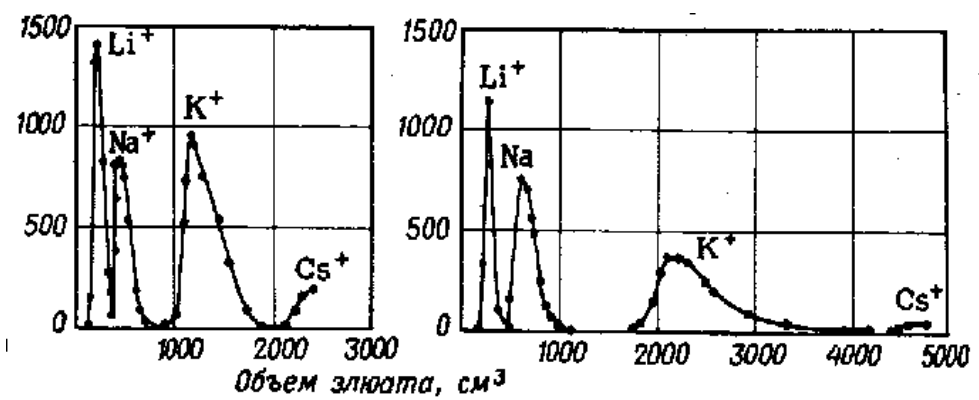

Рис. 2. Разделение ионов щелочных металлов методом ионообменной хроматографии на смоле Wofatit KPS при скорости подачи элюента (0.2 М соляной кислоты) $1 \mathrm{~cm}^{3} /$ мин.

Слева выходная кривая для содержания в катионообменнике дивинилбензола 16\%, справа $26 \%$.

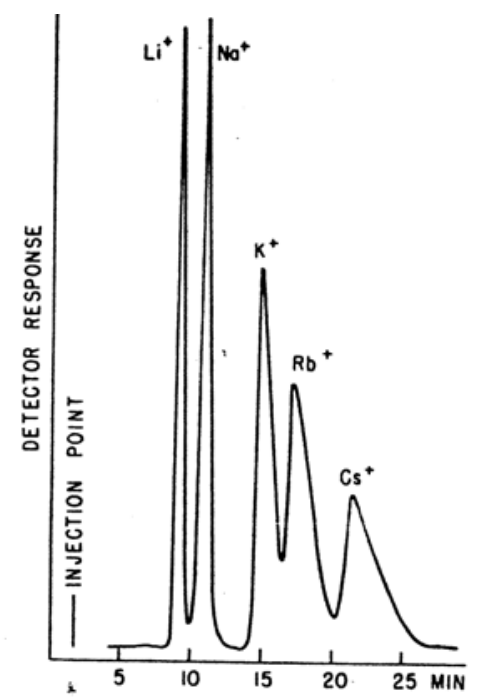

Рис. 3. Разделение катионов щелочных металлов методом ионной хроматографии [8].

Результат, полученный методом ионной хроматографии, показывает, что для эффективного разделения растворов смеси катионов щелочных металлов было достаточно 25 минут. Расчет по уравнению (3) показывает увеличение количества информации при переходе от классической ионообменной хроматографии к ионной хроматографии в 2 тысячи раз при содержании дивинилбензола в катионообменнике 16: и в 4 тысячи раз при содержании в катионообменнике $24 \%$.

Главной причиной увеличения скорости хроматографирования, а, следовательно, и количества информации является уменьшением толщины сорбента в грануле при жидкостной хроматографии. Это относится не только к ионообменной хроматографии, и её высокоэффективному варианту - ионной хроматографии, но к любому варианту высокоэффективной жидкости хроматографии (HPLC). Так как лимитирующей стадией сорбции определяется в большинстве случаев диффузия, то в соответствие с законом Фика плотность потока тем больше, чем меньше толщина слоя сорбента. Существует два варианта уменьшения толщины сорбента. Первый состоит в том, что в использовании гранул коллоидной дисперсности, что принято назвать в настоящее время нанотехнологией. Однако в этом случае уменьшением размера гранул приводит к увеличению коэффициента гидравлического сопротивления колонки и снижению скорости процесса. Второй вариант предусматривает поверхностную модификацию инертных гранул (рис.4). Гранула сорбента может быть 
однородной, как показано на рис.4 слева, и инертной, поверхность, которой модифицирована сорбентом (справа). Второй вариант имеет преимущество в том, что гидравлическое сопротивление значительно меньше, протекаемость выше, что приводит к увеличению скорости хроматографирования при меньшем избыточном давлении.

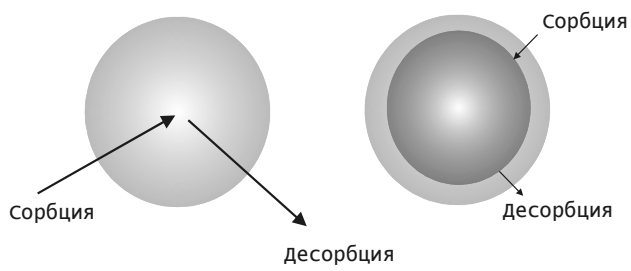

Рис. 4. Схема стандартного сорбента (слева) и инертной гранулы, модифицированной сорбентом (справа).

На практике приходится оценивать не только относительное количество информации, но и рентабельность, которая определяется соотношением

$$
R=\frac{I}{P},
$$

где $R$ - рентабельность, $P$ - общая стоимость анализа, включающие затраты на приборы, реактивы и оплату труда аналитиков.

\section{Заключение}

В аналитической химии в недавнем прошлом широко обсуждалась проблема отсутствия общей теории. Гравиметрия, титриметрия, спектроскопия, хроматография, экстракция и электрохимические методы анализа столь существенно различаются между собой, что создание объединенной теории казалось невозможным. В настоящее время теория информации также не является теорией, способной интегрировать все многообразие аналитических методов. Однако не исключена возможность дальнейшего развития общей теории информации на её основе. Опыт использования теории информации в кибернетике, физике и биологии показывает, что количество информации становится существенной характеристикой даже при описании таких сложных проблем как биологическая эволюция.

Статья отражает основное содержание доклада, сделанного автором на Третьем съезде аналитиков России.

\section{Список литературы/References}

1. Ebeling W., Engel A., Feistel R. Physik der Evolutionsprozesse. Academie-Verlag, Berlin, 1990. $371 \mathrm{p}$.

2. Kaiser H., In. Proc.XIV. Collqu. Spectrosc. Imternat. Debrezen, 1967, A.3, pp. 77.

3. Shannon C.E., Bell. System Tech.J., 1948, Vol. 27, pp. 370-423, 623-656.

4. Doerffel K., Hildebrandt W., Wiss. Z. Tech. Hochsch. Leuna-Merseburg, 1969, Bd. 11, зpp. 30.

Шапошник Владимир Алексеевич - д.Х.н., профессор, химический факультет Воронежского государственного университета, Воронеж
5. Eckschlager K., Collect. Czech. Chem. Commun, 1971, Bd.36, pp. 3016.

6. Danzer K., Z. Chem, 1973, Bd. 13, pp. 20.

7. Eckschlager K., Danzer K. Information Theory in Analytical Chemistry, 1994, John Wiley\&Sons, $263 \mathrm{p}$.

8. Marhol M. Ion Exchangers in Analytical Chemistry. Academia, Prague, 1982. 545 p.

9. Small H., Stevens T., Bauman W., Anal. Chem., 1975, Vol. 47, No 11, pp. 1801-1809.

Shaposhnik Vladimir A. - Dr. Sci.,Professor, Department of Chemistry, Voronezh State University, Voronezh, e-mail: v.a.shaposhnik@gmail.com 